\title{
Chromosome elimination and in vivo haploid production induced by Stock 6-derived inducer line in maize (Zea mays $\mathbf{L}$.)
}

\author{
Zili Zhang · Fazhan Qiu $\cdot$ Yongzhong Liu • \\ Kejun Ma $\cdot$ Zaiyun Li $\cdot$ Shangzhong Xu
}

Received: 17 June 2008/Revised: 27 July 2008/Accepted: 24 August 2008/Published online: 20 September 2008

(C) The Author(s) 2008. This article is published with open access at Springerlink.com

\begin{abstract}
In vivo haploid production induced by inducer lines derived from Stock 6 is widely used in breeding program of maize (Zea mays L.), but the mechanisms behind have not yet been fully understood. In this study, average frequency of haploid induction in four inbred lines by Stock 6-derived inducer line HZI1 was above $10 \%$. About $0.2 \%$ kernels from the cross Hua24 $\times$ HZI1 had mosaic endosperm showing yellow shrunken parts from Hua24 to normal parts with purple aleurone from HZI1. Individual lagged chromosomes and micronuclei were observed in mitotic cells of ovules pollinated by HZI1. Above $56.4 \%$ of the radicles from the kernels with purple aleurone and colorless embryos were mixoploid $(2 n=9$ 21 ), and more than $45.22 \%$ cells were haploid cells $(2 n=10)$ in three crosses. More than $62.5 \%$ of the radicles from the kernels with purple aleurone and purple embryos were mixoploid $(2 n=9-21)$ having $54.27 \%$ cells with $2 n=20$. SSR analysis showed that all haploids from the cross Hua24 $\times$ HZI1 shared the same genomic compositions as Hua24 except for plants Nos. 862 and 857 with some polymorphic DNA bands. The results revealed that chromosome elimination after fertilization caused the haploid production in maize.
\end{abstract}

Communicated by K. Toriyama.

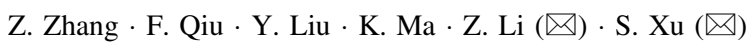

National Key Laboratory of Crop Genetic Improvement,

National Center of Crop Molecular Breeding Technology,

College of Plant Science and Technology,

Huazhong Agricultural University,

430070 Wuhan, People's Republic of China

e-mail: lizaiyun@mail.hzau.edu.cn

S. Xu

e-mail: shangzhongxu@gmail.com
Keywords Zea mays L. - In vivo haploid induction · Mixoploid · Chromosome elimination · SSR

\section{Introduction}

It takes 7-8 generations to obtain a maize inbred line and the plants were still not $100 \%$ pure in traditional breeding. Doubled-haploid (DH) technology improves breeding efficiency by generating inbred lines with $100 \%$ purity and genetic uniformity in just two generations. DH lines make it easy to carry genetic studies and shorten the breeding time significantly. Maize (Zea mays L.) is a typical diploid plant $(2 n=20)$, but haploid individuals $(2 n=10)$ occur naturally at a rate of one per 1,000 kernels (Chase 1949). Maize haploid can be derived either through tissue culture technique (in vitro) or through genetic induction (in vivo). Anther culture in maize is a highly complex and expensive method with low plantlet regeneration rate, which is dependent on genetic background (Beckert 1994; Shatskaya et al. 1994) and is greatly limited for the application in breeding programs. Nowadays, in vivo haploid induction by inducer lines is widely used by most breeders for its high frequency, simple operation and inexpensiveness.

There are two modes of in vivo haploid induction in maize, leading to maternal and paternal haploids, respectively. The genomes of maternal haploids originate from the maternal parent and those of paternal haploids come from the paternal parent. Coe (1959) discovered a haploid inducer, Stock 6, and 1-2\% of the progeny was maternal haploids when it was used as pollinator. Kermicle (1969) obtained $0-2 \%$ paternal haploids when the W23 indeterminate gametophyte (ig) line was used as female parent. However, the rate of haploids obtained with Stock 6 and 
W23 ig was still low and depended on the genotypes (Lashermes and Beckert 1988). The haploid-inducing capacity of the inducer can be increased by selection (Sarkar et al. 1972). To date, a number of new inducers with higher haploid-induction rate have been created (Eder and Chalyk 2002), such as WS14 (Lashermes et al. 1988), ZMS (Chalyk 1994), KMS (Tyrnov and Zavalishina 1984), MHI (Eder and Chalyk 2002), and RWS (Röber et al. 2005).

The mechanism of in vivo haploid induction has not yet been full understood until now (Eder and Chalyk 2002; Röber et al. 2005). Several hypotheses have been put forward to explain the haploid formation through in vivo haploid induction in maize. Firstly, the irregularities during microsporogenesis and fertilization may be involved in haploid induction. $\mathrm{Hu}$ (1990) found the difference between the transmission velocities of two sperms in one microspore, and the sperm with high velocity fertilized normally, while the one with low velocity missed the fertilization, which broke the normal double fertilization and developed the kernels with a haploid embryo (Enaleeva et al. 1996). Chang (1992) suggested two irregular cases occurring in the maize double fertilization. Primarily, the polar nucleus is fertilized, while the egg cell remains unfertilized and develops into embryos with the cell division of the fertilized polar nucleus. Alternatively, the egg cell is destroyed when the pollen tube is entering into embryo sacs, one of the sperms fertilizes the polar nucleus and other develops into haploid embryos. Bylich and Chalyk (1996) detected pollen grains with a pair of morphologically different sperm nuclei in ZMS inducer line, as a result, one of the sperms can fertilize normally, but another does not. Rotarenco and Eder (2003) detected a more than three time higher rate of heterofertilization when haploid inducer was used as pollinator compared to a normal inbred line. Chalyk et al. (2003) observed above 15\% aneuploidy microsporocyte in the inducer MHI and only about $1 \%$ in two inbred lines used as checks. Briefly, the abnormality in microsporogenesis and fertilization may be the reasons of haploid induction. Coe and Neuffer (2005) also supported this hypothesis. Secondly, chromosome elimination after fertilization might be the major mechanism in maize in vivo haploid induction. Wedzony et al. (2002) studied ovaries of inducer line RWS during the first 20 days after self-pollination. In about $10 \%$ of the embryos, micronuclei of variable size were found in the cytoplasm of every cell of the shoot primordium. Such micronuclei generally were the symbol of chromosome elimination from the cell in subsequent divisions (Kasha and Kao 1970). Fischer (2004) showed that a small proportion (1-2\%) of haploids obtained from the cross between the inducer line and a broad-based sample of breeding materials carried one, seldom several paternal chromosome segments through
SSR markers analysis. It indicated that a small fragment from the inducer genome can be transferred into maternal genome of the haploids (Röber et al. 2005).

In present study, we find that chromosome elimination after fertilization leads to in vivo maize haploid production induced by inducer line HZI1 derived from Stock 6. Cells with variable chromosome numbers appear in the radicles of the $F_{1}$ kernels from crosses with inducer HZI1, and individual lagged chromosomes and micronuclei are observed in the pollinated ovules by HZI1. The $F_{1}$ kernels with mosaic endosperm consisting of normal part with purple aleurone and sweet shrunken part without purple aleurone are also observed.

\section{Materials and methods}

\section{Plant materials}

An inducer line HZI1 derived from Stock 6 with $R-n j(R-n j$ : R-navajo, a domiant gene on chromosome 10 for purple aleurone and purple embryo) and $S h 2$ (the dominant gene of $s h 2$ on chromosome 3 for normal endosperm) was used as male parent, and it has normal endosperm with purple aleurone and purple embryo. Three normal inbred lines were used as female parents: HZ124b, HZ85, HZ141 with $r(r$, recessive gene of $R-n j)$ and $S h 2$, and with the normal endosperm with colorless aleurone and colorless embryo, as well as a sweet corn inbred line Hua24 with $r(r$, recessive gene of $R-n j)$ and $\operatorname{sh} 2(\operatorname{sh} 2$, shrunken2, showing shrunken endosperm), and with shrunken endosperm with colorless aleurone and colorless embryo.

The crosses between the inducer HZI1 and above inbred lines were made in field, respectively, and all $F_{1}$ kernels were harvested by single ear and analyzed separately. We identified haploid kernels by using the system of dominant anthocyanin marker genes (Chase 1969; Nanda and Chase 1966). In this system, the expression of the R-nj gene provided an anthocyanin pigmentation of the embryo and the endosperm. Kernels with purple aleurone and colorless embryo were putative haploids and the kernels with purple aleurone and purple embryo were hybrid kernels. The kernels with colorless aleurone and purple embryo were defect kernels and the kernels with colorless aleurone and colorless embryo were from pollen contamination.

\section{Cytology}

Twenty radicles of each parents and 50 radicles of each phenotype of F1 kernels from the crosses of HZI1 as male parent with Hua24, HZ124b, HZ141, and HZ85, were collected after germination for three days. In addition, 17 
radicles of each phenotype of $F 1$ kernels of the cross HZ85 $\times$ HZI1 collected at 3, 5, 7 days after germination respectively. All the collected radicles were used to determine chromosome numbers.

The radicles preparations followed the methods reported by Kindiger and Beckett (1983) except that the collected radicles were pretreated with $2 \mathrm{mM} 8$-hydroxyquinoline for $5 \mathrm{~h}$ at $22^{\circ} \mathrm{C}$. Chromosome numbers were determined following the procedure by $\mathrm{Li}$ et al. (1995). One hundred ovaries of Hua24 $36 \mathrm{~h}$ after pollinated by HZI1 were collected and fixed in a solution of 3:1 ethanol:acetic acid for $24 \mathrm{~h}$ then transferred to fresh solution and stored at $-20^{\circ} \mathrm{C}$ for cytological observations.

Morphology and fertility investigation

The inducer and inbred lines were planted in the field at Wuhan in spring and Hainan Island in winter in 2006. The seedlings after radicles collection were transplanted in the field in spring of 2007 for molecular and morphological investigation. The putative haploid plants from morphological observation obtained from the cross Hua24 $\times$ HZI1 were transplanted adjacent to the female plants Hua24 to facilitate the traits comparison in field, and several traits were measured, such as plant height, ear height, ear leaf length, ear leaf width, tassel length and branches etc., as described by Augers et al. (2004). The data were analyzed by $t$ test (Excel 2003) and clustered using SAS V8.02 (SAS Institute Inc. Cary, NC, 1991-2001).

The pollen fertility was determined as the percentage of pollen grains stained with $1 \% \mathrm{KI} / \mathrm{I}_{2}$. More than 400 pollen grains were counted for each plant. Female inflorescence fertility of haploid plants was determined by the seed setting after pollinated by inbred line Hua24.

\section{SSR analysis}

Maize genomic DNA was isolated from expanding leaves of plants according to CTAB procedure (Saghai-Maroof et al. 1984). SSR analysis was made in the haploid plants of the crosses Hua24 $\times$ HZI1 and HZ124b $\times$ HZI1 and both their parents, by the protocol of Qiu et al. (2007), and clustering was made using NTsys Version2.0. Sequences of all SSR markers were obtained from the MaizeGDB database (http://www. maizegdb.org/ssr.php).

\section{Results}

Frequency, morphology and fertility of haploids

The frequency of haploid induction by HZI1 was averagely above $10 \%$ identified by the $R-n j$ genetic markers in
Table 1 Haploid frequencies identified by the expression of $R-n j$ gene in four crosses

\begin{tabular}{lcc}
\hline Crosses & Total kernels & Average haploid $(\%)$ \\
\hline Hua24 × HZI1 & 880 & 12.65 \\
HZ124b × HZI1 & 2,182 & 11.91 \\
HZ141 × HZI1 & 1,976 & 8.19 \\
HZ85 $\times$ HZI1 & 909 & 13.89 \\
\hline
\end{tabular}

kernels, which ranged from 8.19 to $13.89 \%$ for different female parents (Table 1). Remarkably, about $0.2 \%$ kernels in the $F_{1}$ kernels from the cross Hua24 $\times$ HZI1 had mosaic endosperm of purple aleurone half and yellow shrunken half without purple aleurone (Fig. 1c), for the kernels of Hua24 had shrunken endosperm with colorless aleurone (Fig. 1a), while the kernels of inducer HZI1 had normal endosperm with purple aleurone (Fig. 1b). The mosaic kernels had different sizes of sweet shrunken endosperm in the top centre of the kernels (Fig. 1d1, d2, d3).

Compared with the diploid plants, haploid seedlings of Hua24 were short in stature and grew slowly with distinctive narrow leaves, slim and weak stems and lightly yellow leaves (Chase 1964) (Fig. 1e1, e2). After flowering, there were significantly differences between haploid and diploid plants in plant height, ear height, ear leaf length, ear leaf width, tassel length (Table 2; Fig. 1). The florets of the haploid tassels did not open or rarely contained pollen grains after opening (Fig. 1f3).

The morphological clustering analysis of 21 haploid plants and 22 Hua24 plants showed that all 43 plants were mainly classified into two types, haploid and diploid (data not shown). Haploid No. 857 was clustered into a new type, which might correspond to its morphology. Its tassel was different from other haploid plants by showing the red floret and some pink anthers and less tassel branches (Fig. 1f4). The length of its ear leaf was $37 \mathrm{~cm}$, much shorter than the average $(56 \mathrm{~cm})$ of other haploid plants $(t=32.76, \operatorname{Pr}>|t|,<0.0001)$, the width was $3.7 \mathrm{~cm}$, narrower than the average $(6.5 \mathrm{~cm})$ of others $(t=19.52$, $\operatorname{Pr}>|t|,<0.0001)$, and the tassel branches was 5, significantly fewer than the average 20.7 of others $(t=16.40$, $\operatorname{Pr}>|t|,<0.0001)$.

The pollen fertility of the haploid plants from the cross Hua24 $\times$ HZI was $0-40 \%$ (Fig. 2b1, b2) except for $41 \%$ of No. 858 and $42 \%$ of No. 867 . No seeds were produced by all haploids after pollinated by Hua24, except for 13 seeds from plant No. 857 and 3 seeds from plant No. 858.

Twenty-five haploid plants from the cross HZ124b $\times$ HZI1 were morphologically very similar and had the same phenotype as the female HZ124b. 
Fig. 1 Morphology of haploids from the cross Hua24 $\times$ HZI1. a, b The kernels of the sweet corn inbred line (a), HZI1 (b). c The mosaic endosperm kernel with half normal endosperm and half with sweet shrunken endosperm. Bar $0.5 \mathrm{~cm}$. d1, d2, d3 The mosaic endosperm kernels with different portions of the sweet shrunken endosperm in the ear (arrows). Bar $0.5 \mathrm{~cm}$. e1, e2 Morphology of the haploid plants from Hua24 $\times$ HZI1 cross and Hua24 in the field. e1 Young haploid (left), diploid (right) plants. Bar $10 \mathrm{~cm}$. e2 Adult haploid (left) and diploid (right) plants. Bar $90 \mathrm{~cm}$. f1-f4 Tassels of Hua24 (f1), HZI1 (f2),

haploid No. 858 (f3), No. 857 (f4). Bar $10 \mathrm{~cm}$
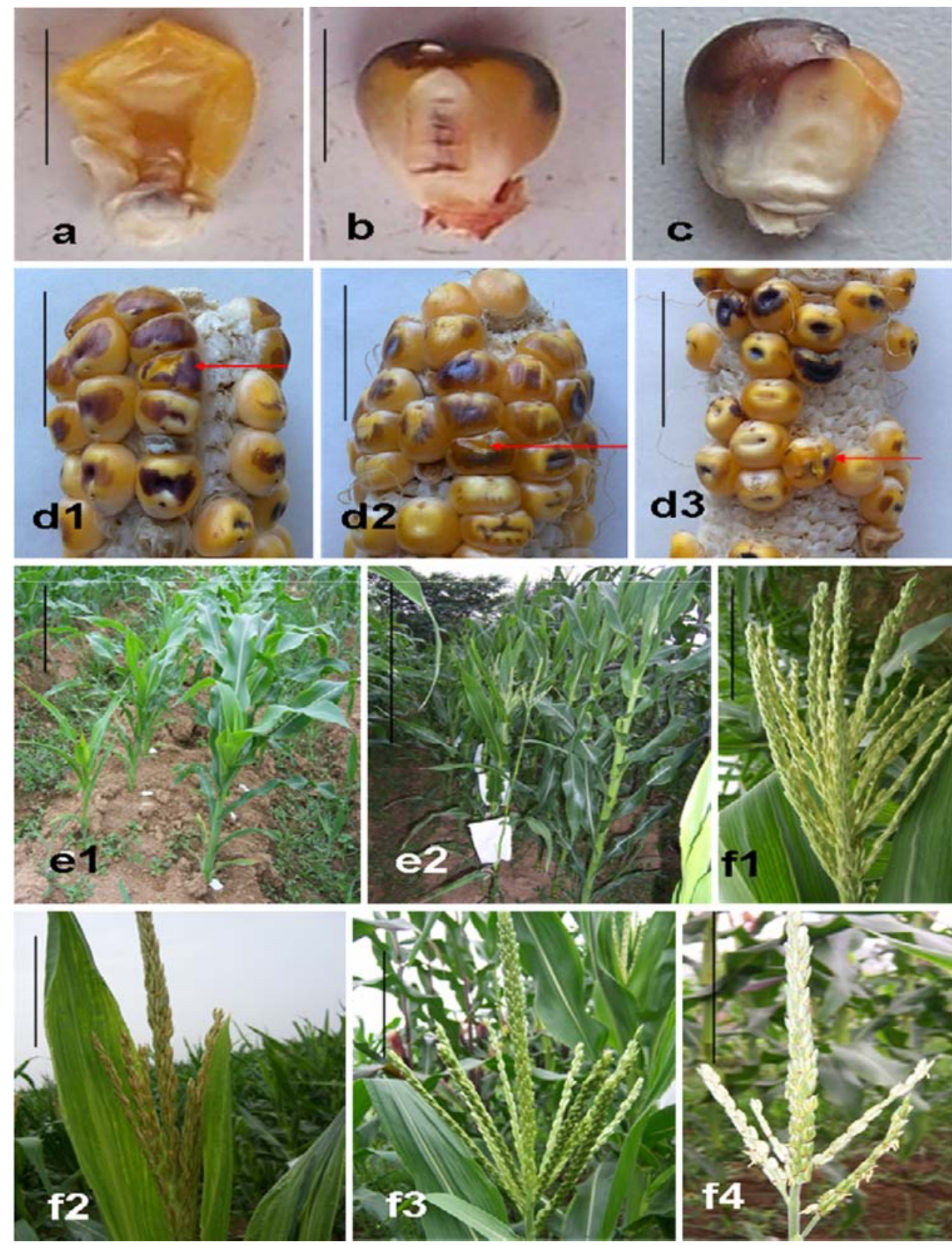

Table 2 Morphology of the haploid plants from cross Hua24 $\times$ HZI1

\begin{tabular}{|c|c|c|c|c|c|c|c|c|}
\hline \multirow[t]{2}{*}{ Morphological traits } & \multicolumn{3}{|c|}{ Haploid } & \multicolumn{3}{|l|}{ Hua24 } & \multirow[t]{2}{*}{$t$ value } & \multirow[t]{2}{*}{$\operatorname{Pr}>|t|$} \\
\hline & Mean & SE & SD & Mean & SE & SD & & \\
\hline Plant height $(\mathrm{cm})$ & 114.14 & 2.31 & 10.59 & 175.38 & 2.81 & 13.78 & -16.82 & $<0.0001$ \\
\hline Ear height $(\mathrm{cm})$ & 39.05 & 1.90 & 8.70 & 88.88 & 1.95 & 9.56 & -18.31 & $<0.0001$ \\
\hline Ear leaf length $(\mathrm{cm})$ & 55.94 & 1.11 & 5.08 & 77.08 & 0.98 & 4.80 & -14.29 & $<0.0001$ \\
\hline Ear leaf width $(\mathrm{cm})$ & 6.37 & 0.19 & 0.87 & 8.85 & 0.13 & 0.62 & -10.85 & $<0.0001$ \\
\hline Tassel length (cm) & 19.73 & 0.70 & 3.19 & 30.42 & 0.58 & 2.84 & -11.78 & $<0.0001$ \\
\hline Tassel branches & 19.95 & 1.18 & 5.40 & 23.79 & 0.81 & 3.97 & -2.69 & 0.0108 \\
\hline
\end{tabular}

$S D$ standard deviation, $S E$ standard error 
Fig. 2 Cytology of $F_{1}$ kernels from Hua24 × HZI1 cross. a1a4 Root-tip metaphases in the radicles of the $F_{1}$ kernels, with $2 n=20$ (a1), 15 (a2), 13 (a3), 10 (a4). Bar $10 \mu \mathrm{m}$. c One lagged chromosome in root metaphase. $\mathbf{d}-\mathbf{g}$ Ovule cells of Hua24 pollinated by HZI1. Bar $10 \mu \mathrm{m}$. d One chromosome lagged at metaphase. e One chromosome lagged at anaphase. f Laggards at telophases. b1, b2 Pollen grains of haploids Nos. 869, 867. Bar $20 \mu \mathrm{m}$ telophase. g1, g2 Micronuclei at
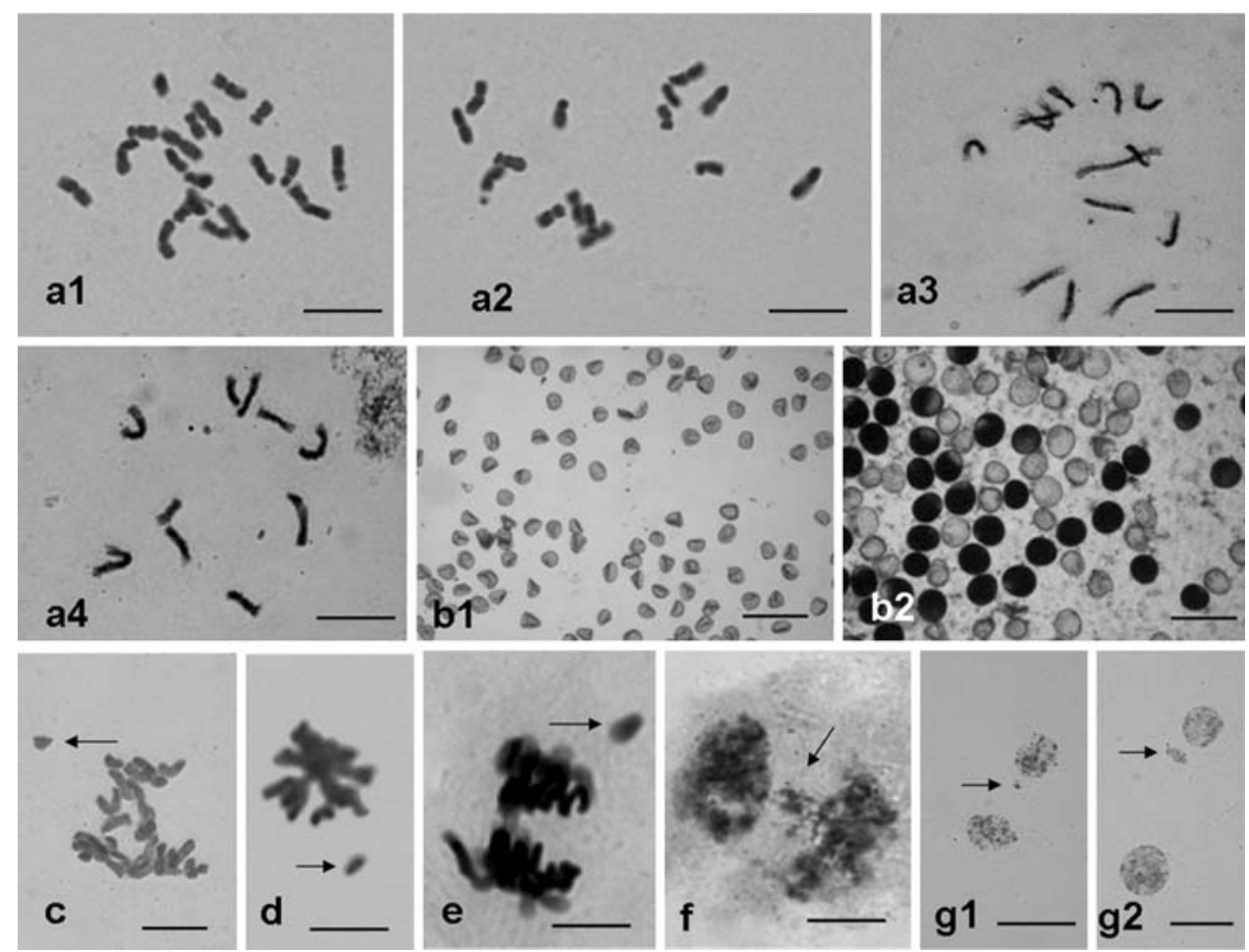

Cytology of $F_{1}$ radicles and pollinated ovules

Chromosome numbers in the female parents HZ85 and

Hua24 and inducer HZI1

In radicles of normal inbred line HZ85, $96.70 \%$ cells had the expected chromosome numbers $2 n=20$ and $3.30 \%$ had $2 n<20$ (Table 3). In Hua24, 96.85\% cells had $2 n=20$ and $3.15 \%$ had $2 n<20$ (Table 3). For inducer HZI1, only $85.41 \%$ cells had $2 n=20$ and $14.59 \%$ had $2 n<20$ but $9.75 \%$ had $2 n=10$, the number of haploid cells. Among the radicles of HZI1, $25 \%$ had $2 n=20$ in all cells, $10 \%$ had $2 n=10$ in majority of cells, and $65 \%$ had $2 n=20$ as most frequent in their cells. So HZI1 can produce haploids in the selfed progeny.
In the radicles of $F_{1}$ kernels with purple aleurone and colorless embryo and $F_{1}$ kernels with purple aleurone and purple embryos obtained from the crosses Hua2 $4 \times$ HZI1, HZ124b $\times$ HZI1, HZ141 $\times$ HZI1, and HZ85 $\times$ HZI1, the cells with different chromosome numbers $(2 n=9-21)$ were observed, with $2 n=10$, or 20 being most frequent in different roots (Table 4). In the radicles of $F_{1}$ kernels with purple aleurone and colorless embryo, the frequencies of cells with $2 n=10$ were $67.47,76.74$ and $45.22 \%$ in crosses of HZI1 with Hua24, HZ124b, and HZ141, respectively. But in the cross HZ85 $\times$ HZI1, the frequency

Table 3 Different chromosome numbers in the radicle cells of cross parents

\begin{tabular}{|c|c|c|c|c|c|c|c|c|c|c|c|c|}
\hline \multirow[t]{2}{*}{ Lines } & \multicolumn{11}{|c|}{ Cells with chromosome numbers } & \multirow[t]{2}{*}{ Total } \\
\hline & 10 & 11 & 12 & 13 & 14 & 15 & 16 & 17 & 18 & 19 & 20 & \\
\hline \multicolumn{13}{|l|}{ HZ85 } \\
\hline Cell no. & 0 & 1 & 2 & 0 & 0 & 1 & 0 & 1 & 2 & 1 & 343 & 351 \\
\hline$(\%)$ & 0.00 & 0.29 & 0.57 & 0.00 & 0.00 & 0.29 & 0.00 & 0.29 & 0.57 & 0.29 & 96.70 & \\
\hline \multicolumn{13}{|l|}{ Hua24 } \\
\hline Cell no. & 0 & 0 & 0 & 0 & 0 & 0 & 1 & 1 & 1 & 2 & 150 & 155 \\
\hline$(\%)$ & 0.00 & 0.00 & 0.00 & 0.00 & 0.00 & 0.00 & 0.63 & 0.63 & 0.63 & 1.26 & 96.85 & \\
\hline \multicolumn{13}{|l|}{ HZI1 } \\
\hline Cell no. & 51 & 2 & 2 & 2 & 4 & 2 & 5 & 1 & 2 & 5 & 447 & 523 \\
\hline$(\%)$ & 9.75 & 0.38 & 0.38 & 0.38 & 0.76 & 0.38 & 0.98 & 0.19 & 0.38 & 0.98 & 85.41 & \\
\hline
\end{tabular}


Table 4 Numbers and percentages of radicle cells in two types of embryos of $F_{1}$ kernels from four crosses

\begin{tabular}{|c|c|c|c|c|c|c|c|c|c|c|c|c|c|c|c|c|}
\hline \multirow[t]{2}{*}{ Crosses } & \multirow[t]{2}{*}{ Radicles } & & \multicolumn{13}{|c|}{ Number and percentage of cells with chromosome number } & \multirow[t]{2}{*}{ Total } \\
\hline & & & 9 & 10 & 11 & 12 & 13 & 14 & 15 & 16 & 17 & 18 & 19 & 20 & 21 & \\
\hline \multirow[t]{4}{*}{ Hua24 × HZI1 } & I & Number & 8 & 253 & 49 & 10 & 22 & 4 & 0 & 4 & 2 & 5 & 1 & 17 & 0 & 375 \\
\hline & & $(\%)$ & 2.13 & 67.47 & 13.07 & 2.67 & 5.87 & 1.07 & 0.00 & 1.07 & 0.53 & 1.33 & 0.27 & 4.53 & 0.00 & \\
\hline & II & Number & 7 & 22 & 13 & 8 & 5 & 4 & 8 & 12 & 4 & 13 & 9 & 127 & 2 & 234 \\
\hline & & $(\%)$ & 2.99 & 9.40 & 5.56 & 3.42 & 2.14 & 1.71 & 3.42 & 5.13 & 1.71 & 5.56 & 3.85 & 54.27 & 0.85 & \\
\hline \multirow[t]{4}{*}{ HZ124b $\times$ HZI1 } & I & Number & 5 & 165 & 15 & 5 & 4 & 3 & 2 & 0 & 2 & 2 & 2 & 10 & 0 & 215 \\
\hline & & $(\%)$ & 2.33 & 76.74 & 6.98 & 2.33 & 1.86 & 1.40 & 0.93 & 0.00 & 0.93 & 0.93 & 0.93 & 4.65 & 0.00 & \\
\hline & II & Number & 2 & 5 & 4 & 4 & 4 & 4 & 7 & 7 & 7 & 7 & 12 & 123 & 2 & 188 \\
\hline & & $(\%)$ & 1.06 & 2.66 & 2.13 & 2.13 & 2.13 & 2.13 & 3.72 & 3.72 & 3.72 & 3.72 & 6.38 & 65.43 & 1.06 & \\
\hline \multirow[t]{4}{*}{ HZ141 × HZI1 } & I & Number & 29 & 194 & 13 & 8 & 19 & 4 & 5 & 5 & 6 & 13 & 21 & 111 & 5 & 429 \\
\hline & & $(\%)$ & 6.8 & 45.22 & 3.03 & 1.86 & 4.43 & 0.93 & 1.17 & 1.17 & 1.39 & 3.03 & 4.89 & 25.87 & 1.17 & \\
\hline & II & Number & 4 & 8 & 6 & 6 & 5 & 8 & 5 & 14 & 9 & 13 & 26 & 139 & 5 & 248 \\
\hline & & $(\%)$ & 1.61 & 3.23 & 2.42 & 2.42 & 2.02 & 3.23 & 2.02 & 5.65 & 3.63 & 5.24 & 10.48 & 56.05 & 2.02 & \\
\hline \multirow[t]{4}{*}{ HZ85 × HZI1 } & I & Number & 18 & 193 & 17 & 7 & 6 & 15 & 15 & 16 & 16 & 31 & 24 & 329 & 4 & 691 \\
\hline & & $(\%)$ & 2.60 & 27.93 & 2.46 & 1.01 & 0.87 & 2.17 & 2.17 & 2.32 & 2.32 & 4.49 & 3.47 & 47.61 & 0.58 & \\
\hline & II & Number & 12 & 27 & 14 & 15 & 12 & 17 & 22 & 17 & 22 & 47 & 69 & 391 & 7 & 672 \\
\hline & & $(\%)$ & 1.79 & 4.02 & 2.08 & 2.23 & 1.79 & 2.53 & 3.27 & 2.53 & 3.27 & 6.99 & 10.27 & 58.18 & 1.04 & \\
\hline
\end{tabular}

I, Radicles of kernels with purple aleurone and colorless embryo; II, radicles of kernels with purple aleurone and purple embryo

of the cells with $2 n=10$ was $28 \%$, while that of those with $2 n=20$ was $47.60 \%$. In the radicles of $\mathrm{F}_{1}$ kernels with purple aleurone and purple embryo from the four crosses, the cells with $2 n=20$ were $54.27,65.43,56.05$, and $58 \%$, respectively.

More than half (56.4\%) of the radicles of $\mathrm{F}_{1}$ kernels with purple aleurone and colorless embryo had variable chromosome numbers $(2 n=9-21)$ and the kernels were classified into 6 categories: I, haploid, $2 n=10$ in all cells; II, mixoploid haploid, $2 n=10-15$ with $2 n=10$ of most frequent; III, mixoploid haploid, $2 n=10-20$ with $2 n=10$ of most frequent; IV, mixoploid diploid, $2 n=10-20$ with $2 n=20$ of most frequent; V, mixoploid, $2 n=11-19$; VI, diploid, with $2 n=20$ in all cells (Table 5). Types I and II were more frequent in crosses Hua24 $\times$ HZI1, HZ124b $\times$ HZI1, while types II and IV in crosses HZ141 $\times$ HZI1, HZ85 $\times$ HZI1. In crosses Hua24 $\times$ HZI1, HZ124b $\times$ HZI1, percentages of haploid radicles were 45 and $32 \%$, respectively, but only 16.67 and $2.23 \%$ in crosses HZ141 × HZI1, HZ85 × HZI1.

The kernels with purple aleurone and purple embryo had $62.5 \%$ radicles with different chromosome numbers $(2 n=9-21)$ and were put into five types: I, diploid, $2 n=20$ only; II, mixoploid diploid, $2 n=10-20$ with $2 n=20$ being most frequent; III, mixoploid diploid, $2 n=16-20$ with $2 n=20$ being most frequent; IV, mixoploid haploid, $2 n=10-20$ with $2 n=10$ being most frequent; V, mixoploid, $2 n=11-19$ (Table 5). Types I, II and III were more frequent in all four crosses. The percentages of diploid kernels in four crosses were 37.5, $16.12,20$, and $12.5 \%$, respectively (Table 5 ).

In ovule cells from the cross Hua24 $\times$ HZI1, individual chromosomes were arranged out of equatorial plate in metaphase cell (Fig. 2d) and lagged at anaphase/telophase cells (Fig. 2e, f). Micronuclei were formed (Fig. 2g1, g2).

Change of cells with different chromosome numbers in radicles at three times

In the radicles of the kernels with purple aleurone and colorless embryos from the cross HZ85 $\times$ HZI1 collected 3, 5, 7 days after germination, the frequencies of the cells with $2 n=10$ increased from 13.29 , to 53.88 , to $70 \%$, while those of $2 n=20$ decreased from 65.19 , to 20.87 , to $10 \%$ (Fig. 3a). In the radicles of the kernels with purple aleurone and purple embryos at three times, the frequencies of the cells with $2 n=10$ and 20 varied at much narrower ranges and cells with $2 n=20$ were predominant (about $50 \%$ ); the percentages of $2 n=20$ are 59.32, 57.32, $48.13 \%$, respectively; and those of $2 n=10$ are 5.08, 1.22 , $5.00 \%$, respectively (Fig. 3b).

SSR analysis

SSR analysis using 28 markers on haploid plants from the cross Hua24 $\times$ HZI1 showed that all haploids shared the same genomic compositions as the female Hua24 except two (Nos. 857 and 862) (Fig. 4a, b). Haploid No. 857 plant 
Table 5 Numbers and frequencies of the kernels with different chromosome numbers in two types of embryos from four crosses i, Radicles of kernels with purple aleurone and colorless embryo

ii, Radicles of kernels with purple aleurone and purple embryo

\begin{tabular}{|c|c|c|c|c|c|c|}
\hline Crosses & Phenotype & Type & $\begin{array}{l}\text { Most frequent one } \\
\text { and ranges of } \\
\text { chromosome numbers }\end{array}$ & Plant number & $(\%)$ & Total \\
\hline \multirow[t]{11}{*}{ Hua24 × HZI1 } & \multirow[t]{6}{*}{$\mathrm{i}$} & I & 10 & 18 & 45.00 & \multirow[t]{6}{*}{40} \\
\hline & & II & $10 ; 10-15$ & 14 & 35.00 & \\
\hline & & III & $10 ; 10-20$ & 3 & 7.50 & \\
\hline & & IV & $20 ; 10-20$ & 2 & 5.00 & \\
\hline & & $\mathrm{V}$ & $11-19$ & 3 & 7.50 & \\
\hline & & VI & 20 & 0 & 0.00 & \\
\hline & \multirow[t]{5}{*}{ ii } & I & 20 & 15 & 37.50 & \multirow[t]{5}{*}{40} \\
\hline & & II & $20 ; 10-20$ & 18 & 45.00 & \\
\hline & & III & $20 ; 16-20$ & 5 & 12.50 & \\
\hline & & IV & $10 ; 10-20$ & 1 & 2.50 & \\
\hline & & V & $11-19$ & 1 & 2.50 & \\
\hline \multirow[t]{11}{*}{ HZ124b × HZI1 } & \multirow[t]{6}{*}{$\mathrm{i}$} & I & 10 & 8 & 32.00 & \multirow[t]{6}{*}{25} \\
\hline & & II & $10 ; 10-15$ & 14 & 56.00 & \\
\hline & & III & $10 ; 10-20$ & 0 & 0.00 & \\
\hline & & IV & $20 ; 10-20$ & 2 & 8.00 & \\
\hline & & $\mathrm{V}$ & $11-19$ & 0 & 0.00 & \\
\hline & & VI & 20 & 1 & 4.00 & \\
\hline & \multirow[t]{5}{*}{ ii } & I & 20 & 5 & 16.12 & \multirow[t]{5}{*}{31} \\
\hline & & II & $20 ; 10-20$ & 13 & 41.93 & \\
\hline & & III & $20 ; 16-20$ & 13 & 41.93 & \\
\hline & & IV & $10 ; 10-20$ & 0 & 0.00 & \\
\hline & & V & $11-19$ & 0 & 0.00 & \\
\hline \multirow[t]{11}{*}{ HZ141 × HZI1 } & \multirow[t]{6}{*}{$\mathrm{i}$} & I & 10 & 6 & 16.67 & \multirow[t]{6}{*}{36} \\
\hline & & II & $10 ; 10-15$ & 11 & 30.56 & \\
\hline & & III & $10 ; 10-20$ & 2 & 5.56 & \\
\hline & & IV & $20 ; 10-20$ & 13 & 36.11 & \\
\hline & & V & $11-19$ & 2 & 5.56 & \\
\hline & & VI & 20 & 2 & 5.56 & \\
\hline & \multirow[t]{5}{*}{ ii } & I & 20 & 6 & 20.00 & \multirow[t]{5}{*}{30} \\
\hline & & II & $20 ; 10-20$ & 17 & 56.67 & \\
\hline & & III & $20 ; 16-20$ & 5 & 16.67 & \\
\hline & & IV & $10 ; 10-20$ & 0 & 0.00 & \\
\hline & & V & $11-19$ & 2 & 6.67 & \\
\hline \multirow[t]{11}{*}{ HZ85 × HZI1 } & \multirow[t]{6}{*}{$\mathrm{i}$} & I & 10 & 1 & 2.63 & \multirow[t]{6}{*}{38} \\
\hline & & II & $10 ; 10-15$ & 6 & 15.79 & \\
\hline & & III & $10 ; 10-20$ & 1 & 6.67 & \\
\hline & & IV & $20 ; 10-20$ & 19 & 50.00 & \\
\hline & & V & $11-19$ & 7 & 18.42 & \\
\hline & & VI & 20 & 4 & 10.53 & \\
\hline & \multirow[t]{5}{*}{ ii } & I & 20 & 7 & 12.50 & \multirow[t]{5}{*}{56} \\
\hline & & II & $20 ; 10-20$ & 40 & 71.43 & \\
\hline & & III & $20 ; 16-20$ & 7 & 12.50 & \\
\hline & & IV & $10 ; 10-20$ & 0 & 0.00 & \\
\hline & & V & $11-19$ & 2 & 3.58 & \\
\hline
\end{tabular}

from maternal parent and $15 \%$ from paternal parent. The SSR clustering of the haploid plants selected by morphology from the cross Hua24 $\times$ HZI1 showed that all the had $61 \%$ heterozygous bands, $7.1 \%$ from maternal parent, $3.6 \%$ from paternal parent and $3.6 \%$ novel for two parents. Haploid No. 862 had $43 \%$ loci novel for two parents, $30 \%$ 

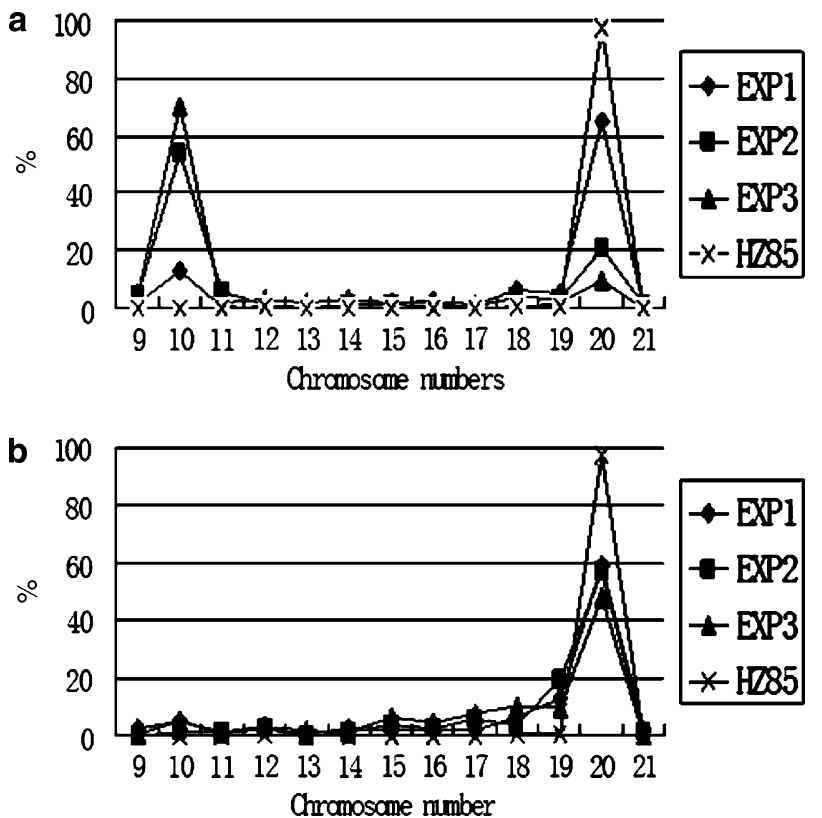

Fig. 3 Frequencies of radicle cells with different chromosomes in $F_{1}$ kernels from the cross HZ85 $\times$ HZI1 at three times. a Kernels with purple aleurone and colorless embryos. b Kernels with purple aleurone and purple embryo. EXP1, EXP1, and EXP3: radicles collected 3 days (EXP1), 5 days (EXP2), 7 days (EXP3) after generation, respectively

P1P2 123456789101112131415161718192021

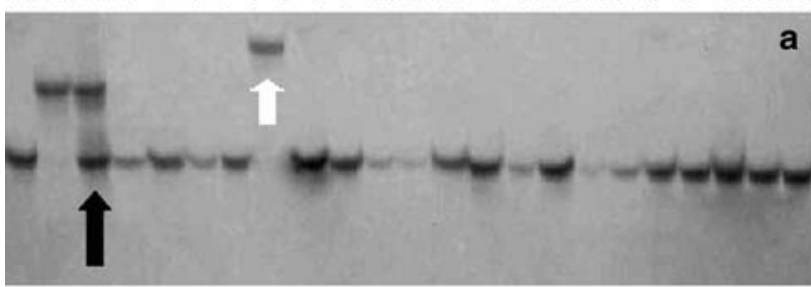

P1 P2 1234556789101112131415161718192021

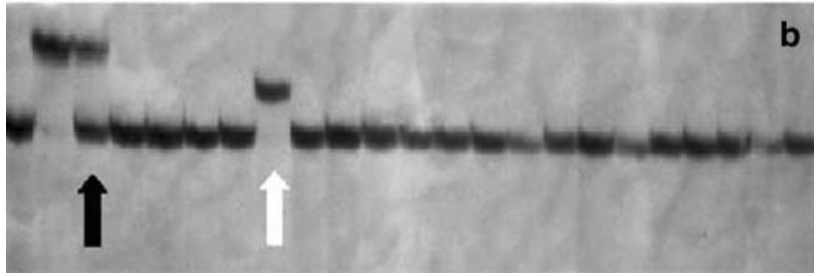

Fig. 4 Representative SSR profiles generated from two markers in the haploid plants from cross Hua24 $\times$ HZI1. a From primer M178 (umc2059). Black arrow shows heterozygous band of haploid No. 857, and white arrow shows one novel band in haploid No. 862. b From primer M219 (bnlg1065). Black arrow shows heterozygous band in haploid No. 857, and white arrow shows novel band in haploid No. 862. P1 Hua24, P2 HZI1

haploid plants were put between the two parents and most haploids were close to the female parent Hua24 except for plants Nos. 862 and 857 (Fig. 5).

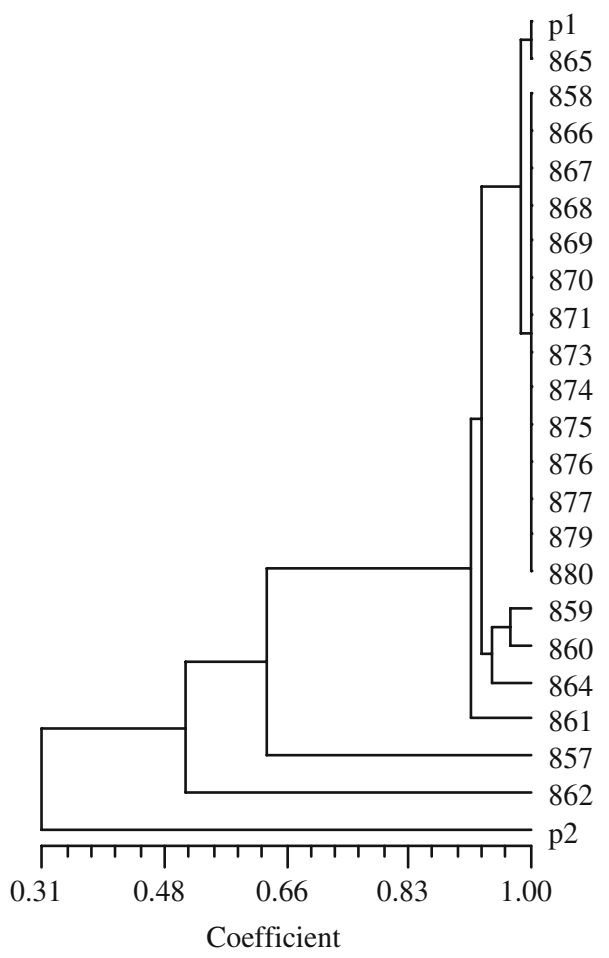

Fig. 5 SSR clustering of haploid plants from Hua24 $\times$ HZI1 cross. p1 Hua24, p2 HZI1

Twenty-five plants from the cross HZ124b $\times$ HZI1 showed the same SSR profiles and shared the same genomic compositions as the female HZ124b.

\section{Discussion}

The frequencies of haploid plants from the kernels with purple aleurone and colorless embryo from the four crosses varied widely from 25.09 to $88.0 \%$ (Table 5), as reported by others (Lashermes et al. 1988; Shatskaya et al. 1994; Chalyk 1999; Eder and Chalyk 2002). Progenies from inducing hybridizations with haploid inducer were investigated for morphology, cytology and genomic compositions, which enable us to identify the haploids efficiently. Most putative haploids screened by $R-n j$ morphological marker were similar with the maternal parent in phenotype. Cytology revealed that some lagged chromosomes and micronuclei occurred in the mitotic cells of fertilized ovules (Fig. 2) and mixoploidy in the radicle of the $F_{1}$ kernels (Tables 4, 5; Fig. 2). Some paternal bands were found in few haploids (Nos. 857 and 862) by SSR analysis (Figs. 4, 5). The kernels with mosaic endosperm were found in the cross Hua24 $\times$ HZI1 (Fig. 1).

Lagged chromosomes (Fig. 2d, e, f) and the micronuclei (Fig. 2g1, g2) were often used as direct evidence of chromosome elimination and haploid production (Kasha and 
Kao 1970), which often occurred in inter-, intra-specific hybridizations in Gramineae and other species (Laurie 1989). Micronuclei were also found in the primordium of the inducer RWS ovaries 20 days after self-pollination (Wedzony et al. 2002). Hence, the results indicate that the chromosome elimination during the early development of the embryo after fertilized by inducers is one of the main causes of the in vivo haploid induction in maize.

About $15 \%$ radicle cells of the inducer HZI1 were aneuploid, while about $3 \%$ in inbred lines. Similar results were also observed for microsporocytes of the inducer MHI and two inbred lines used as checks (Chalyk et al. 2003). So aneuploidy is involved in haploid induction by the inducer and occurred in the microsporocytes and radicle cells of inducers, which showed that the chromosomes of the inducer were prone to lose during mitotic and meiotic divisions and provided the cytological basis for the induction ability to produce haploids in crosses with other lines.

Among six types of $F_{1}$ kernels with purple aleurone and colorless embryos in four crosses, Types I, II, III were haploid plants in the field, showing that the chromosomes from HZI1 were completely lost during the development process of embryos and plants. In $F_{1}$ radicles collected at different times, the frequency of cells $(2 n=10)$ gradually increased, while that of the cells $(2 n=20)$ reduced. Radicles of putative haploid kernels also had some cells with $2 n=20$ (Tables 4,5 ), but the frequency of cells with $2 n=10$ tended to increase (Fig. 3a), leading to the production of haploid plants. This also indicated that the chromosome elimination probably occurred in the whole growth process of the haploid plants.

Diploid cells in the maize haploid tissues were likely due to the doubling of haploid cells (Khokhlov et al. 1976) or the residue of the chromosome elimination. Plants of types IV, V looked like hybrid plants in the field probably for the high percentages of diploid cells in apical meristem of plants, which was known as incorrect selection by the expression of the $R-n j$ gene (Beckert 1994). Kernels of type VI probably did not look like haploids or hybrids, as found by Chalyk et al. (2003). All haploid plants were smaller than the diploid parents (Rotarenco 2000) had low pollen fertility (Shatskaya et al. 1994). Diploid cells in haploid No. 867 of type III were probably from the doubling of haploid cells and resulted in higher pollen stainability (Fig. 2b2). The female spikelet of the haploid plants from the cross Hua24 $\times$ HZI1 developed abnormally and had lower seedsets than that of others (Chalyk 1994), which was possibly affected by the environment and cultivation conditions.

Of five types of kernels with purple aleurone and purple embryo in all four crosses, Types I, II, III were phenotypically hybrid plants, but variations in chromosome numbers were shown by Types II, III. Types IV and V often presented some morphological deviations from hybrid plants, as found by Chalyk et al. (2003), which might be related with their wide ranges of chromosome numbers in cells. The mixoploidy nature of these plants should be the result of partial elimination of chromosomes from HZI1. The morphological traits of haploid No. 857 are different from that of other haploids and female parent Hua24, and some SSR DNA bands from HZI1 indicated the introgression of genetic element from the inducer during the process of chromosome lost, the same results was found by Fischer (2004). Haploid No. 862 with the same morphology as other haploids had $43 \%$ loci novel for two parents, which indicated that No. 862 was not caused by the pollen contamination and the high rate of the novel bands might be attributable to the heterozygosity of HZI1. In mixoploid No. $862(2 n=9-21)$ with high frequency of cells $(2 n=10)$ and SSR loci $(15 \%)$ from the inducer the incomplete chromosomes elimination with some chromosomal rearrangements probably occurred. The SSR and morphology clusterings also separated plants Nos. 862 and 857 from all other haploids (Fig. 5). But the maternal haploids from another cross HZ124b $\times$ HZI1 in morphology and genomic compositions were probably either due to the genotypic effect of different female parents.

In about $0.2 \%$ kernels of $F_{1}$ progeny from the cross Hua24 $\times$ HZI1, endosperms were mosaic (Fig. 1c, d1, d2, d3), while $F_{1}$ hybrid kernels should have normal endosperm with purple aleurone. According to the genotypes of two parents, the mosaic kernels most likely resulted from the elimination of chromosomes carrying $R-n j$ and $S h 2$, because the rate of simultaneous mutation of two genes is very slow [the mutation rates of the $R-n j$ and $S h 2$ genes in maize kernels are about $5 \times 10^{-4}$ and $1.2 \times 10^{-6}$ (Zhu 2002), respectively, and the rate of transpositional insertion is about $10^{-5}$ (Zhu 2002)]. The variable sizes of the shrunken sweet parts (Fig. 1c, d1, d2, d3) in the mosaic endosperm may be attributed to the different elimination times of paternal chromosomes. The mosaic kernels (Fig. 1c) were probably the result of the complete elimination in one daughter cell from the first mitosis of the primary endosperm nuclei. The mosaic kernels in Fig. 1d1, $\mathrm{d} 2, \mathrm{~d} 3$ formed later and the sweet shrunken endosperm often occurred at the top of the endosperm.

Complete and partial elimination of chromosomes from one parent was documented in plant wide crosses (Kasha and Kao 1970; Laurie 1989; Laurie and Bennett 1989; Mochida et al. 2004; Riera-Lizarazu et al. 1996; Gernand et al. 2005, 2006). Chromosome elimination was generally attributed to the unbalanced chromosome sets (Kasha and Kao 1970). The chromosome elimination mechanism behind in vivo haploid induction in maize may be similar to that of wide hybridizations or be caused by some genetic factors in the inducers (Lashermes and Beckert 1988; Deimling et al. 1997; Barret et al. 2008), which need to 
elucidate in future and may be important for the selection of inducers with high frequency.

Open Access This article is distributed under the terms of the Creative Commons Attribution Noncommercial License which permits any noncommercial use, distribution, and reproduction in any medium, provided the original author(s) and source are credited.

\section{References}

Augers DL, Ream TS, Birchler JA (2004) A test for a metastable epigenetic component of heterosis using haploid induction in maize. Theor Appl Genet 108:1017-1023

Barret P, Brinkmann M, Beckert M (2008) A major locus expressed in the male gametophyte with incomplete penetrance is responsible for in situ gynogenesis in maize. Theor Appl Genet (in press)

Beckert M (1994) Advantages and disadvantages of the use of in vitro/in situ produced DH maize plants. BAJAJ YPS Biotech Agri Forest 25:201-213

Bylich VG, Chalyk ST (1996) Existence of pollen grains with a pair of morphologically different sperm nuclei as a possible cause of the haploid-inducing capacity in ZMS line. Maize Genet Coop Newslett 70:33

Chalyk ST (1994) Properties of maternal haploid maize plants and potential application to maize breeding. Euphytica 79:13-18

Chalyk ST (1999) Creating new haploid-inducing lines of maize. Maize Genet Coop Newslett 73:53-54

Chalyk S, Baumann A, Daniel G, Eder J (2003) Aneuploidy as a possible cause of haploid-induction in maize. Maize Genet Coop Newslett 77:29

Chang MT (1992) Preferential fertilization induced from Stock 6. Maize Genet Coop Newslett 66:99-100

Chase SS (1949) Monoploid frequencies in commercial double cross hybrid maize, and in its component single cross hybrids and inbred lines. Genetics 34:328-332

Chase SS (1964) Monoploids and diploids of maize: a comparison of genotypic equivalents. Am J Bot 51:928-933

Chase SS (1969) Monoploids and monoploid-derivatives of maize (Zea mays L.). Bot Rev 35:117-167

Coe EH (1959) A line of maize with high haploid frequency. Am Nat 93:381-382

Coe EH, Neuffer MG (2005) Darker orange endosperm color associated with haploid embryos: Y1 dosage and the mechanism of haploid induction. Maize Genet Coop Newslett 79:7

Deimling S, Röber FK, Geiger HH (1997) Methodik und genetic der in-vivo- haploideninduktion bei mais. Vortr PXanzenzüchtg 38:203-224

Eder J, Chalyk S (2002) In vivo haploid induction in maize. Theor Appl Genet 104:703-708

Enaleeva NK, Tyrnov VS, Selivanova LP, Zavalishina AN (1996) Single fertilization and the problem of haploidy induction in maize. Doklady Biol Sci 353:225-226

Fischer E (2004) Molekulargenetisvhe untersuchungen zum vokommen paternaler DNA-ubertragung bei der in-vivo-haploideninduktion bei mais (Zea mays L.). PhD Dissertation. University of Hohenheim, Grauer Verlag, Stuttgart

Gernand D, Rutten T, Pickering R, Houben A (2006) Elimination of chromosomes in Hordeum vulgare $\times H$. bulbosum crosses at mitosis and interphase involves micronucleus formation and progressive heterochromatinization. Cytogenet Genome Res 114:169-174

Gernand D, Rutten T, Varshney A, Rubtsova M, Prodanovic S, Brüß C, Kumlehn J, Matzk F, Houben A (2005) Uniparental chromosome elimination at mitosis and interphase in wheat and pearl millet crosses involves micronucleus formation, progressive heterochromatinization, and DNA fragmentation. Plant Cell 17:2431-2438

Hu S (1990) Male germunit and sperm heteromorphism: the current status. Acta Bot Sin 32:230-240

Kasha KJ, Kao KN (1970) High frequency haploid production in barley (Hordeum vulgare L.). Nature 225:874-876

Kermicle JL (1969) Androgenesis conditioned by a mutation in Maize. Sci 166:1422

Khokhlov SS, Tyrnov VS, Grishina EV, Davoyan NI (1976) Haploidy and breeding, Nauka, Moscow, 221

Kindiger BK, Beckett JB (1983) Modified root tip squash technique. Maize Genet Coop Newslett 57:32-33

Lashermes P, Beckert M (1988) Genetic control of maternal haploidy in maize (Zea mays L.) and selection of haploid inducing lines. Theor Appl Genet 76:405-410

Lashermes P, Gaihard A, Beckert M (1988) Gynogenetic haploid plants analysis for agronomic and enzymatic markers in maize (Zea mays L.). Theor Appl Genet 76:570-572

Laurie DA (1989) The frequency of fertilization in wheat $\times$ pearl millet crosses. Genome 32:1063-1067

Laurie DA, Bennett MD (1989) The timing of chromosome elimination in hexaploid wheat $\times$ maize crosses. Genome 32:953-961

Li Z, Liu HL, Luo P (1995) Production and cytogenetics of intergeneric hybrids between Brassica napus and Orychophragmus violaceus. Theor Appl Genet 91:131-136

Mochida K, Tsujimoto H, Sasakuma T (2004) Confocal analysis of chromosome behavior in wheat $\times$ maize zygotes. Genome 47:199-205

Nanda DK, Chase SS (1966) An embryo marker for detecting monoploids of maize (Zea mays L.). Crop Sci 6:213-215

Qiu F, Zheng Y, Zhang Z, Xu S (2007) Mapping of QTL associated with waterlogging tolerance during the seedling stage in maize. Ann Bot 99:1067-1081

Riera-Lizarazu O, Rines HW, Phillips RL (1996) Cytological and molecular characterization of oat $\times$ maize partial hybrids. Theor Appl Genet 93:123-135

Röber FK, Gordillo GA, Geiger HH (2005) In vivo haploid induction in maize-performance of new inducers and significance of doubled haploid lines in hybrid breeding. Maydica 50:275-283

Rotarenco VA (2000) Synchronization of cell cycles as a means of enhancing the efficiency of chromosome doubling in maize. Maize Genet Coop Newslett 74:13-14

Rotarenco VA, Eder J (2003) Possible effect of heterofertilization on the induction of maternal haploids in maize. Maize Genet Coop Newslett 77:30

Saghai-Maroof MA, Soliman KM, Jorgensen RA, Allard RW (1984) Ribosomal DNA spacer-length polymorphisms in barley: Mendelian inheritance, chromosomal location, and population dynamics. Proc Natl Acad Sci USA 81:8014-8018

Sarkar KR, Panke S, Sachan JKS (1972) Development of maternalhaploidy-inducer lines in maize (Zea mays L.). Indn J Agric Sci 42:781

Shatskaya OA, Zabirova ER, Shcherbak VS, Chumak MV (1994) Mass induction of maternal haploids in corn. Maize Genet Coop Newslett 68:51

Tyrnov VS, Zavalishina AN (1984) Inducing high frequency of matroclinal haploids in maize. Dokl Akad Nauk SSSR 276:735738

Wedzony M, Röber FK, Geiger HH (2002) Chromosome elimination observed in selfed progenies of maize inducer line RWS. In: XVIIth International Congress on Sex Plant Rep. Maria CurieSklodowska University Press, Lublin, p 173

Zhu J (2002) Genetics, 3rd edn. The Publishing House of the Chinese Agriculture, Beijing, p 250 